\title{
Parameters and modes of the technological process of scattered rice sowing
}

\author{
Ivan Masienko*, Sergey Shevchenko, and Kirill Glytyan \\ Kuban State Agrarian University named after I.T.Trubilin, 350044, Krasnodar, Russia
}

\begin{abstract}
The paper presents the determination of main parameters and operating modes of scattered centrifugal devices of technical units when using them in the technology with scattered sowing when performing the technological operation of sowing rice in liquid-flexible soil. Theoretical studies of the dependence of design features of the blade of a spreading disk on the number of blades installed on it and the radius of curvature of the end bend on the height of blades are carried out. The values of the diameter of the disk of a centrifugal dispenser and parameters of blades are set, which will increase the uniformity of distribution of seed material for a given width of a gripper and reduce its consumption.
\end{abstract}

Krasnodar Territory is the main rice-producing region of Russia. Every year, Kuban produces more than $80 \%$ of rice produced in the country. Rice is one of the high-yielding grain crops and with the use of modern technologies, its yield can be 7-8 t/ ha and reach up to 10 $\mathrm{t} / \mathrm{ha}$. A significant disadvantage in rice production is rice sowing. This is due to the fact that the process of rice sowing has specific features and differs significantly from the sowing of grain crops [1,2]. There are two technologies of rice growing in Krasnodar Territory: technology with the "dry" processing of checks and the technology of making checks filled with water ("water").

The technology of rice cultivation "on dry land" is quite well developed and is widely used in rice farms of the region, which cannot be said about the technology of rice cultivation "on dry land", which is a relatively new and promising technology, but has a number of shortcomings that need to be addressed soon.

The advantage of the technology of rice cultivation by water with scattered rice sowing is that regardless of the climatic conditions, this technology is applicable in any zone of the region. The number of field preparation operations does not exceed 3-4. Scattered rice sowing leads to a significant reduction in the sowing time, which affects the maturation time of rice. There is also an increase in the yield of 8-13\% compared to the "dry" rice cultivation technology. Labor costs are reduced by 4.5 people / ha compared to the basic technology $[3,4]$.

The disadvantage of this technology is the implementation of rice sowing in a scattered way, in which there is a violation of agricultural requirements imposed on this operation, which is in ensuring the uniform distribution of seeds over the entire width of the capture, which is reflected in increased consumption of seed material, uneven sowing, and a de-

\footnotetext{
${ }^{*}$ Corresponding author: ivan.masienko@yandex.ru
} 
crease in the yield of main products. The identified shortcomings lead to the need to study and determine main parameters and operating modes of scattered centrifugal devices for further development of technical units, when using them in the technology with scattered sowing, when performing the technological operation of sowing rice in liquid-flexible soil.

In the technology of rice cultivation with processing of checks filled with water, rice sowing can be carried out with the seeder-disperser SNC-500, mounted on a tractor MTZ82 and equipped with a sealing trail. For scattered rice sowing, mounted machinesdispersers of mineral fertilizers of domestic and foreign production, such as RMU-500, RUM-1000, Amazone ZA-TS 4200 ProfisHydro, Strumyk S500 are also often used in the design of which single-disk and double-disk blade seeding units are installed. An approximate diagram of these units is shown in Figure 1.

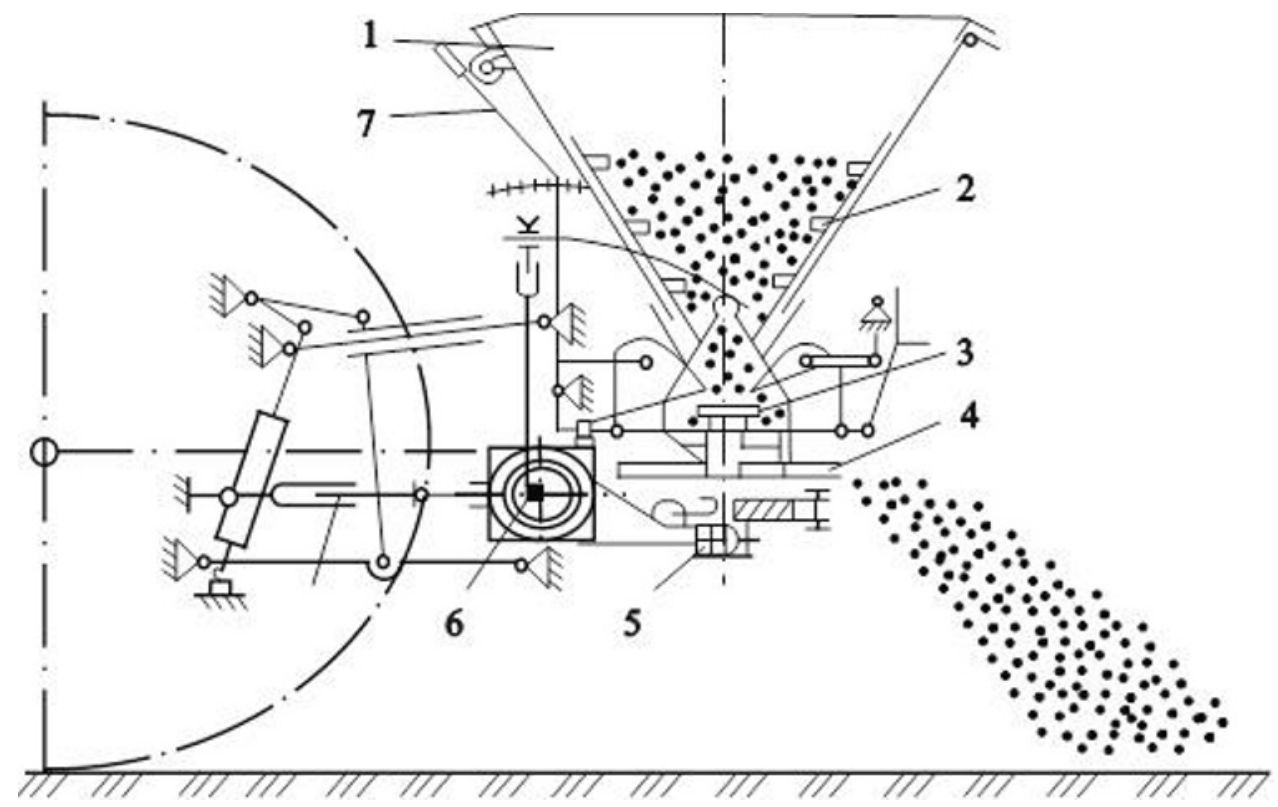

1-hopper for seed material; 2-vaulter; 3-dosing device;

4-disks rotating towards each other; 5-gearbox; 6-rotor; 7-regulator.

Fig. 1. Diagram of a seeding machine analogous to a centrifugal seed disperser

The seeder SNC-500 is designed for scattered sowing of rice, the design of which consists of main components: a trapezoidal hopper, in the lower part of which a dosing device is installed, through which the sowing material is fed to two plates rotating towards each other. In the design of the disk there are grooves, along which the seeds slip when it rotates. The seeding material under the action of centrifugal force is distributed over the surface of the check for a given width of a gripper.

The disadvantage of using the SNC-500 seeder is that seeds sown are scattered at different speeds, and it is not possible to achieve the same uniformity of sowing. Also, with this method of sowing, rice seeds fall to different depths, which leads to uneven germination. The advantages of this method of sowing are the high productivity of the unit.

The main disadvantage of sowing rice in checks filled with water is the uneven sowing of seeds and the different depth of their embedding. Therefore, we proposed to make changes to the design of a seeder-disperser, which will allow the sowing of rice to achieve a uniform distribution of seeds on the surface of the check. 
The researches of scientists of Kuban State Agrarian University named after I. T. Trubilin determined that the main condition for the high-quality operation of the centrifugal device of the seeding machine, which ensures the uniformity of seeding, is an orderly flight of seeds (particles) from blades.

There is a violation of the ordered harvest when the seeds of rice seed material are reflected from the blades of the dispersing disk. Due to the impact of seeds on the surface of the blade, they are reflected and have a different range of flight in the initial angle of scattering.

To exclude the reflection of seeds from the working edge of the blade, it is necessary to sharpen it accordingly. Previously, scientists established the value of the maximum possible angle of sharpening of the working edge, which should not exceed $15^{\circ}[5,6]$. Reflection is excluded due to the fact that the relative velocity vector coincides with the reflected surface. Any kind of impact on the reflected surface is excluded with this direction of the relative velocity of the particle (Figure 2).

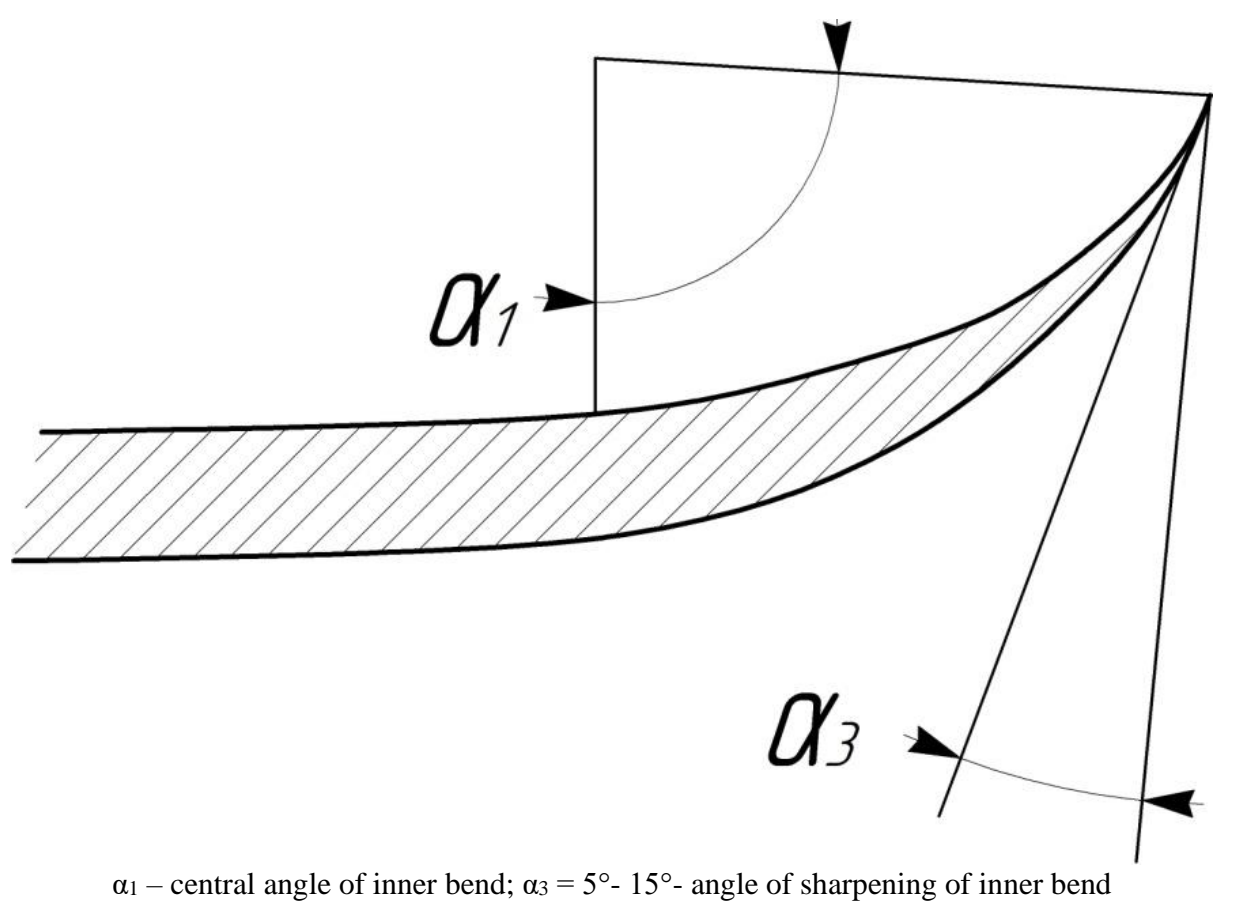

Fig. 2. Angle of working edge of end bending of the blade

Reflection from the disk plane is excluded when the material is captured by the blade on the fly. At the same time, spillage of the material over the edge of the working area of the blade should be completely excluded. Therefore, the volume of material enclosed in the end bend of the blade is limited by the height. The path traversed by the material to capture the blade "fly-in" is equal to the height of the latter.

We derive the formula by transformations that will allow us to determine the feed of sown material by a dispersing machine:

$$
Q=0,00182 r_{q} \alpha h_{0} r_{a} p n k_{1},
$$

We accept the initial requirements for further transformations: $z_{L}-$ amount of blade mounted on a seeding disk, $n$ - frequency of rotation of the seeding disk. Then we get an expression for determining the mass of seeding material per one blade of the disk: 


$$
q_{L}=\frac{Q}{z_{L} n}
$$

Substituting the expression for determining the feed (1), we derive the expression for determining the mass of seeding material per blade:

$$
q_{L}=\frac{0,00182 r_{q} \alpha h_{0} r_{a} p k_{1}}{z_{L}}
$$

The maximum volume of material enclosed in the receiving part of the inner bend is a part of the cylinder. The base of the latter is a sector bounded by a central angle $\alpha_{1}$ with a radius of curvature $r_{l}$.

Taking into account the initial data, the expression for determining the volume of the marked cylinder will have the form:

$$
V=0,00873 r_{L}^{2} \alpha_{1} h
$$

Determine the mass of seed material according to the expression:

$$
P_{л}=0,00873 r_{L}^{2} \alpha_{1} h \rho k_{0},
$$

where $r_{L}$ - radius of curvature of the inner bend of the blade, $\mathrm{m}$;

$k_{0}$ - coefficient that takes into account the presence of gaps and pores;

$\rho$ - bulk weight of seed material, $\mathrm{kg} / \mathrm{m}^{3}$;

$h$ - disk blade height, m;

The condition for grabbing the seed material with the blade of the disk on fly should look like:

$$
P_{L}>q_{\pi}
$$

In this case, the vector of the relative velocity of particles is parallel to circumferential velocity of the working edge of the end bend of the blade has the expression:

$$
0,00873 r_{\pi L}^{2} \alpha_{1} h \rho k_{0}>\frac{0,00182 r_{q} \alpha h_{0} r_{a} p k_{1}}{z_{L}}
$$

Changing the obtained expression, we determine the radius of curvature of the inner bend of the blade, taking into account the accepted value of the central angle of the inner bend $\alpha_{1}=80^{\circ}[7,8,9,10]$.

$$
r_{L}=\sqrt{\frac{0,0026 r_{q} \alpha h_{0} r_{a} k_{1}}{h z_{L} k_{0}}}
$$

Figure 3 shows the dependence of the radius of curvature of the internal bend of the blade on the number of blades installed on the disk. 


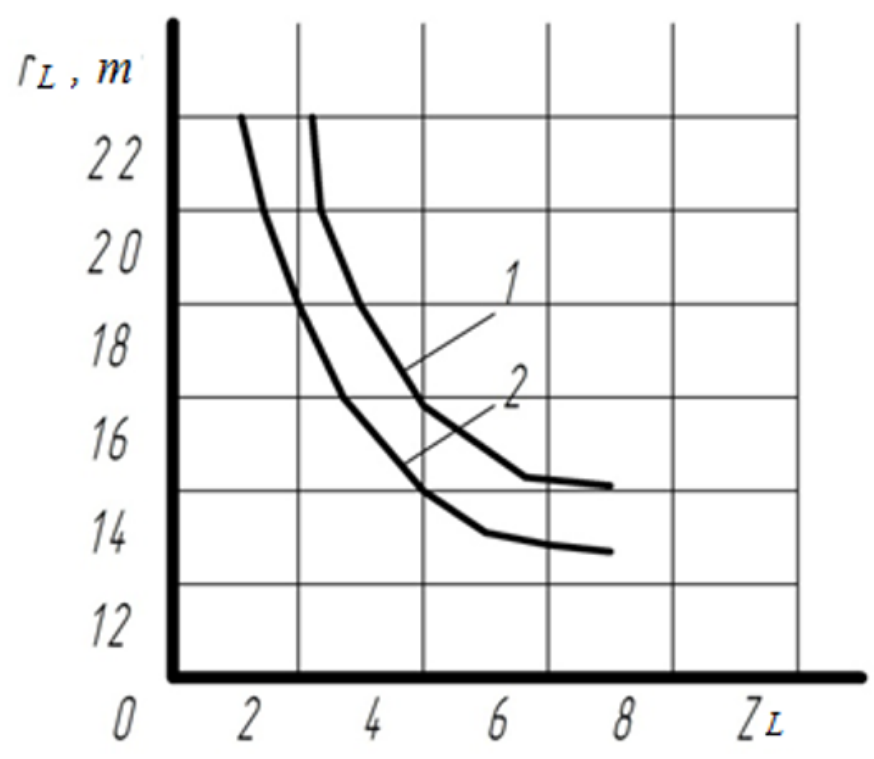

1 -feed to the disk $0.8 \mathrm{~kg} / \mathrm{s} ; 2$-feed to the disk $0.6 \mathrm{~kg} / \mathrm{s}$

Fig. 3. Dependence of the radius of curvature of the internal bend on the number of blades

Analyzing the obtained data of the dependence graph, we can conclude that the radius of curvature of the internal bend decreases with an increase in the number of blades installed on the spreading disk with a constant supply of seed material. The minimum radius of curvature of the internal bend is observed in the design of the spreading disk with six blades.

Figure 4 shows the dependence of the radius of curvature of the end bend on the height of blades.

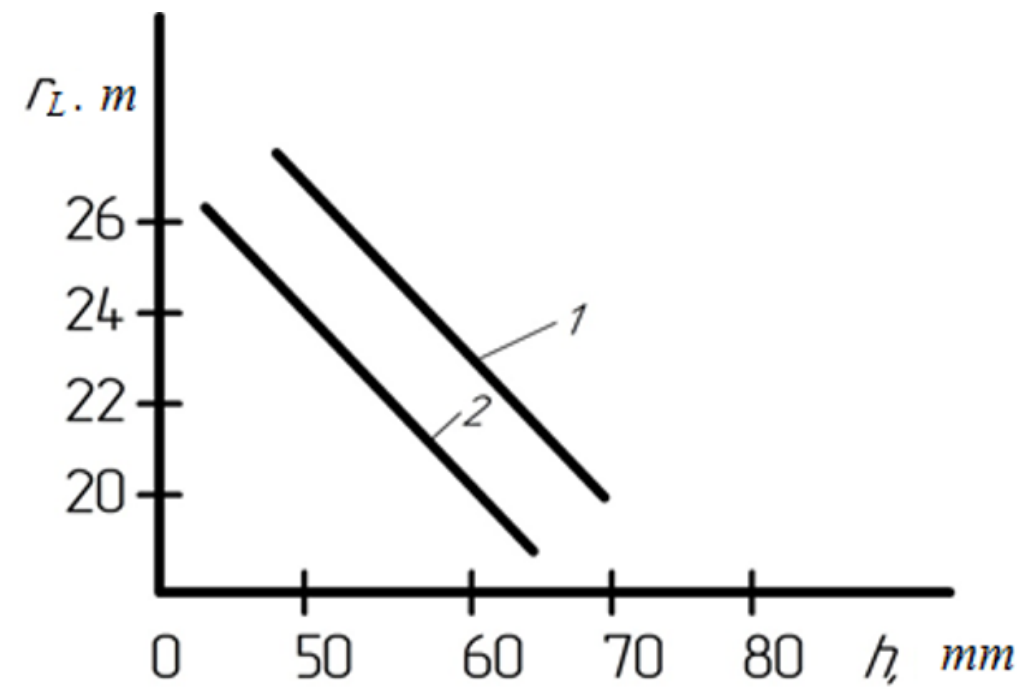

1-feed to the disk $0.8 \mathrm{~kg} / \mathrm{s}$; 2 -feed to the disk $0.6 \mathrm{~kg} / \mathrm{s}$

Fig. 4. Dependence of the radius of curvature of the end bend on the height of the blade 
Analyzing Figure 4, it can be concluded that the radius of curvature of the internal end bend decreases with increasing blade height.

For further development of designs of seed machines of the spreading action it is necessary to take into account the following parameters: in the design of a centrifugal spreader, a flat disk with a diameter of $500 \mathrm{~mm}$ with six radially fixed rectangular blades, measuring $140 \mathrm{~mm} \times 60 \mathrm{~mm}$, should be installed. Each blade is equipped with an internal bend with a sharpening angle of no more than $15^{\circ}$ and a radius of curvature equal to $14 \mathrm{~mm}$. The set values of design parameters of the elements of the centrifugal spreader will increase the uniformity of seed material distribution for a given width of a gripper and reduce its consumption.

\section{References}

1. I. Masienko, Theoretical determination of the harvester propulsion type for rice harvesting, I. Masienko, I.Grigoryan, S. Shevchenko. IOP Conference Series: Earth and Environmental Science, 403(1), p. 012100, (2019)

2. I. Masienko, Patent analysis of design features of non-cereal crop choppers, I. Masienko, A.Vasilenko, S. Schvchenko, E3S Web of Conferences, 193, 01029, (2020)

3. 3V.Konovalov, Analytical study of design parameters of the grinding unit of disk harrows, V. Konovalov, S. Konovalov, V. Igumnova. IOP Conference Series: Earth and Environmental Science, 403, 012086, (2019)

4. V. Konovalov, Analysis of the directions of the development of mechanization units for processing the neartrunk area in the garden, V. Konovalov, S. Konovalov, E3S Web of Conferences, 193, 01013, (2020)

5. A. Karabanitsky, On the issue of reflection of fertilizer particles by blades of a centrifugal disk, In proceedings of Kuban State Agricultural Institute, 176 (204),p.37-45, (1979)

6. A. Karabanitsky, Relationship between design parameters and operating modes of a centrifugal device of mineral fertilizer spreaders. In collection of articles of Kuban State Agricultural Institute, 103 (131), p. 77-82, (1975)

7. V.Konovalov,Justification of design parameters of a disk working body with a changing radius of curvature, V. Konovalov, E3S Web of Conferences, 193, 01014, (2020)

8. V. Konovalov, Constructive-technological diagram of the rotary-string cultivator and the definition of its main parameters, V. Konovalov, S. Konovalov, V. Igumnova, E3S Web of Conferences, 126, 00039, (2019)

9. I.Masienko, Development prospects of mobile rice straw crushers, Всборнике: E3S Web of Conferences, 126, p. 00022, (2019)

10. V.Karpenko, Innovation process of machine harvesting of peas and its efficiency, Karpenko V., Masienko I., Eranova L.B, In collection of articles: E3S Web of Conferences, 126, p. 00021, (2019) 